\title{
Optical Microscopy Has Evolved To Overcome Limitations in Resolution and Image Clarity, (An Overview of Some Available Options)
}

\author{
Marcus O’Reilly
}

\begin{abstract}
Optical microscopy has served the Biological Sciences as an invaluable tool for visualization of very small structures. Researchers in the biological sciences can harness recent improvements in optical microscopy to provide new insights in their own fields. The limitation that light diffraction places on optical resolution has been met by exploiting Extreme Ultra Violet [EUV] Radiation but this has also introduced new challenges. The problem posed by out of focus light from points outside the focal plane has been met by the development of Light Sheet Fluorescent Microscopy which also helps minimize photo-toxicity in living cells. Some of the options available to researchers to exploit these advances are discussed.
\end{abstract}

Keywords - EUV Radiation, Light sheet fluorescent microscopy, Optical microscopy.

\section{OVERCOMING THE LIMITATION IMPOSED BY LIGHT DIFFRACTION.}

Lord Raleigh's investigations into the optical resolution limit [1] demonstrated that once maximal numerical aperture was reached, resolution could only be improved by a reduction of the working wavelength. Thus the pursuit of greater resolution in microscopes has focused on reduction of their working wavelengths, from wavelengths in the visible spectrum to the shorter wavelengths of ultra violet, to deep ultraviolet (DUV), and extreme ultraviolet (EUV) and regions of the $\mathrm{X}$ ray spectrum. EUV wavelengths range from 10 to $121 \mathrm{~nm}$ [2] however technological advances have been exploited to allow the use wavelengths below 10nm [3]. The properties of EUV wavelengths require that significant obstacles must be overcome when they are used in optical microscopy.

Surface absorption of these wavelengths is a significant obstacle, which is overcome by the use of advanced multilayer reflective optics when focusing/manipulating these wavelengths [4].

Conventional optical microscopy exploits refraction, however the optical properties of EUV wavelengths rule out significant phase shift without loss of image detail, thus reflection and diffraction are used to manipulate and focus these wavelengths [5].

Marcus O'Reilly, University College Dublin, Ireland.
Wavelength (nm)

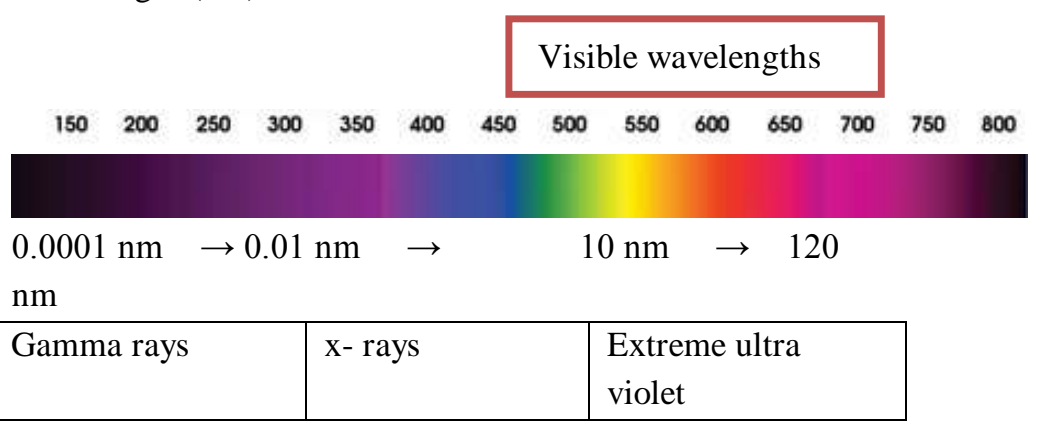

Fig. 1 Electromagnetic spectrum.

Effectively meeting the diffraction properties required to manipulate ever decreasing wavelengths also presents a challenge since it requires corresponding reductions in the choice of diffraction apparatus. Thus 'state of the art' nanofabrication technology, in the form of electron beam lithography is required to effectively meet the demands of the half pitch of these diffractive structures [6]. Decreasing dimensions in turn place far greater accuracy demands on line placement during manufacture of the Fresnel Zone plates used to meet the diffraction demands of EUV microscopes.

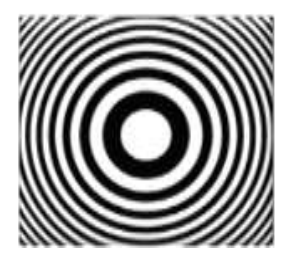

Fig. 2 Fresnel Zone plate

High-resolution electron beam lithography techniques have been developed to meet the demands placed on pattern generation in terms of accuracy of calibration and alignment [5]. Successful Nanofabrication of diffractive components using this technology places critical demands in four key areas of the manufacturing process; placement of the electron beam; accuracy of axis calibration; sub-pixel image processing of overlays and smooth production of arc shapes [5]. 
New challenges are introduced by the very nature of the lens-less imaging system adopted in EUV microscopy. Reflectometry has been referred to as a cornerstone of EUV microscopy [7] with multi-layered ellipsoidal mirrors employed to focus the EUV on the object [8]. EUV Microscopy depends on the use of Fresnel zone plate objective (holographic lens) to form the image. Fresnel zone plates are suitable for focusing these wavelengths [8] where they function as holographic lenses that operate by diffraction. A monochromatic light source is a basic requirement of the Fresnel zone plate [9]. Provision of EUV wavelengths of a quality suitable for use in this type of microscopy has also presented a challenge. Synchrotron radiation [10], and incoherent laser-plasma based sources [11] have been employed but 'laser-produced plasma sources' [12] appear to offer the most practical solution particularly in terms of cost while at the same time producing a usable wavelength. Spatial resolution is degraded where object thickness greatly exceeds the microscopes objective focus depth and the focal length depends on the wavelength used [13]. The band width is also critical with regard to spatial resolution [13]. EUV microscopes are highly technique sensitive and the smallest misalignments of any optical component can result in enormous final image aberrations. Thus wavelength control and nano-focusing are important challenges in addition to thermal drift, vibration and other anomalies [14]. This highlights the importance of great precision in manufacturing and assembling each of the component parts of the microscope together with skilled manipulation of the instrument. To eliminate as far as possible all extraneous sources of error it is necessary to house the optical system that constitutes the EUV microscope inside a high efficiency vacuum environment [15].

Despite the technological challenges posed by EUV microscopy it overcomes several of the limitations of optical microscopy due to the superiority of its analytical sensitivity and its high spatial resolution resulting from the much shorter wavelengths used. It is a most useful tool with widespread applications in nanotechnology, inspection of semiconductor circuits and three-dimensional imaging of living cells in aqueous biological fluids with straightforward sample preparations.

Solving the problem presented by out of focus light.

Another development in optical microscopy which extends the role it can play in biological research is Light Sheet Fluorescence Microscopy (LSFM). This enables biologists obtain a three dimensional view of living organisms, and extends the microscopes potential enabling it to analyse developmental, physiological and patho-physiological processes at a cellular level. All technological advancement builds on the work of others in the constant quest for a better understanding and insight into biological processes. LSFM is the present day technological descendant of a concept that had its genesis in the form of the "ultramicroscope" perfected by Richard Adolf Zsigmondy in 1903. The ultramicroscope used white visible light but later when fluorescent illumination was harnessed this technology was referred to as "orthogonal plane fluorescence microscopy" [16] and was used to record detailed 3-dimensional images of rodent cochlear morphology. A decade later 3- dimensional microscopic images of complete living organisms had arrived on the scene [17] and the Single Plane Illumination Microscope (SPIM) was born.

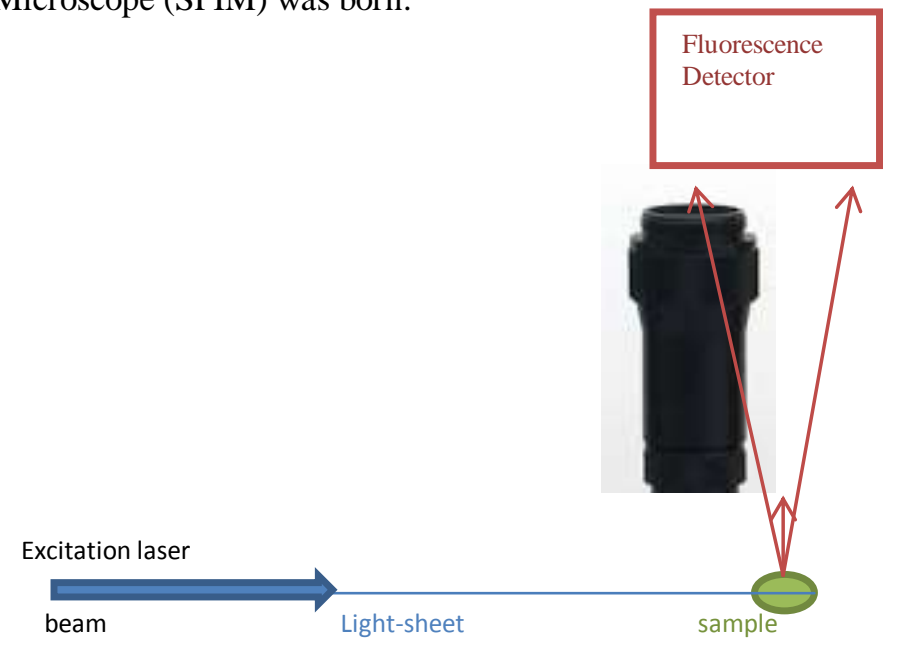

Fig.3 Basic layout of a light sheet microscope

This is an evolving technology and recent improvements in optical resolution have brought it into the super-resolution range of individual molecule localization-SPIM [18] and the harnessing of Bessel beams (incapable of diffraction) in Bessel plane imaging has allowed an axial resolution of $0.3 \mu \mathrm{m}$ with structured illumination [19], [20]. Bessel plane imaging has several different modes each with its own imaging metrics and with its own perspective, and requires settings that are specifically tuned to the properties of the sample under investigation. Physicists are finding novel ways of improving and adapting this technology and it has rapidly evolved into a multi-disciplinary scientific endeavour. The "Lattice light sheet microscope" developed in 2014 [21] by the research team of Eric Betzig, that included Robert Tjian and physicist Steven Chu has brought this technology to a level where it is now possible to physically observe in- vitro molecular events, including DNA replication and repair, RNA transcription etc. They employed extremely thin light sheets from 2-dimensional optical lattices from which 3D images (at diffraction limit and beyond) at sub second time intervals can be captured. The structured system of illumination employed in the lattice light sheet microscope helps in the reduction of out of focus excitation a problem that has plagued light sheet microscopy.

The scientific community has at its disposal a number of DIY Light sheet microscope systems. 


\section{Open SPIM (Open Selective-Plane Illumination Microscopy)}

Open source platforms are available where scientists share technological information enabling anyone to assemble their own light sheet microscopes DIY style together with advice on how best to operate them. By bringing together researchers in this field and harnessing their collaborative efforts these open source platforms aim to drive the evolution of this technology. Their magnanimous approach prompted the Strasbourg based Human Frontier Science Program (HFSP) that aims to encourage international collaboration in basic research, to provided financial backing to Tomancak and Huisken laboratories at the Dresden based Max Planck Institute of Molecular Cell Biology and Genetics to collaborate with the University of Wisconsin-Madison based Laboratory for Optical and Computational Instrumentation, a partnership that resulted in the launching of OpenSPIM.

Open SPIM: [Open Selective-Plane Illumination Microscopy] is an open access platform providing all that is required in terms of hardware and software for the construction of a customizable plane illumination microscope [22]. This platform provides open access via a wiki site (http://openspim.org) where extremely detailed information on the construction of light sheet microscopes is provided. The component parts for the microscope are easily sourced optical components, a charge coupled device (CCD) camera and a laser. Picard industries manufacture a motorized USB 4D stage specifically for use with SPIM which can be used to control motion with great precision plus the sample tubes rotation [23]. The handling of the enormous quantities of data generated in light sheet microscopy requires specialized software and this this is also available free of charge. SPIM recorded data interpretation and handling requires advanced alogarithms which are provided by "Fiji" software available to all from the companion site (http://fiji.sc). These open platform resources combine to allow any laboratory to introduce light sheet microscopy with the minimum of effort and expenditure.

The Dresden based Huisken Lab at the Max Planck Institute of Molecular Cell Biology and Genetics, in an effort to promote this technology has also launched a stand- alone educational initiative to highlight the potential of SPIM, titled "eduSPIM". This compact SPIM is on display as part of the "HiLights" exhibition at the Technische Sammlungen, Dresden, Germany. Visitors including the online visitors to its web-site http://eduspim.org/ can view the vasculature of a zebrafish larva (prepared specimen) using this SelectivePlane Illumination Microscope by selecting any of 7 control buttons.

Another DIY open platform light sheet microscopy system called the "OpenSpinMicroscopy project" has also been launched [24]. The OpenSpinMicroscopy project also makes freely available blueprints on how to build the microscope together with operating instructions and data handling/processing software, through its website (https://sites.google.com/site/openspinmicroscopy/).

Information is provided regarding various LSFM designs including the incorporation of fluidics and flow cytometry. DIY instructions are detailed together with open source hardware based on the "Arundo" prototyping board and the AT mega 328 microcontroller, using micromanagermicroscope control software. The hardware control is an open source plug in, and data handling is based on free "Fiji" and "Micromanager" software.

Taken together the "OpenSpinMicroscopy project"and the "OpenSPIM" platforms both make available detailed and complete LSFM construction plans together with data processing software which allows any laboratory to introduce this technology at minimal expense. Manufacturers of microscopes have not been "idling in the wings" during the emergence of LSFM and many have manufactured this type of microscope.

However, until lately each provided seems to be more suited to "niche" areas of biological research or quality control purposes rather than having widespread applicability in biological research.

\section{Lightsheet Z.1}

The "Lightsheet Z.1" which incorporates a wide selection of illumination wavelengths, is marketed by 'Carl Zeiss Microscopy'. It borrows heavily from the work of pioneering researchers [25] and uses a combination of cylindrical optics and a "beam scattering mechanism" to shape the illuminating light sheet thus allowing illumination of the sample from two sides. The Lightsheet Z.1 incorporates a patented pivot scanner and helps to minimise sample bleaching by the use of low intensity laser illumination. This microscope design is most suited for time-delay fluorescent image capture, of living tissues with data collected over an extended time frame such as for embryogenesis studies. Lightsheet Z.1 Multiview Processing is provided by the 3D VisArt software and they provide two data storage modules; the 8 TB Storage and Data Analysis Module and the 32 TB Storage and Data Analysis Module.

However, until lately each provided seems to be more suited to "niche" areas of biological research or quality control purposes rather than having widespread applicability in biological research.

\section{UltraMicroscope II}

"LaVision BioTec" presently market the "UltraMicroscope II" their second generation light sheet fluorescent microscope [26] an upgraded version of a 2007 design [27]. It is capable of managing sample sizes ranging from a few microns to over a centimetre and unique among commercially available LSFM samples can be imaged in a liquid environment i.e. aqueous buffer or organic solvent. It has 3 working distances; $4 \mathrm{~mm}, 6 \mathrm{~mm}$ and $10 \mathrm{~mm}$. It has 6 thin light sheets allowing a three dimensional image stack of the sample to be generated at cellular resolution, when the sample is moved through these light sheets. It maintains consistent sample illumination by the use of bi-directional sheet excitation. Fluorescent light emitted from the sample is detected by a 
sCMOS equipped microscope positioned at 90 degrees to the illumination plane. The field of view diagonal ranges from $17 \mathrm{~mm}$ down to $1.7 \mathrm{~mm}$, eliminating the need for stitching when the sample size is large. It does have an obvious drawback however in that it lacks multi-view imaging. File formats tiff and the tiff.ome, which must be transferred to commercial 3D processing software solutions such as Imaris, Velocity, Amira or Arivis, or to open source software platforms like ImageJ or Fiji for processing.

Leica-microsystems use the "twinflect mirror" [28] to make a right angled deflection of the illuminating light sheet which allows a full confocal and light sheet microscopy combination in the same instrument, the LeicaTCS SP8 DLS (Digital LightSheet) microscope.

Then we have companies offering components that can be used to custom build your light sheet fluorescent microscop to your own particular requirements by providing various options to suite particular sample properties or to facilitate obtaining specific data. "Applied Scientific Instrumentation" has various components on offer. By using these components a dual- sided or single light-sheet arrangement can be adopted on the inverted microscope basic design [29]. This arrangement is best suited to high throughput screening since it caters for rapid scanning of large conventionally prepared samples on a glass surface.

"Phaseview" market an innovative hardware plus software package that can be used to add light sheet imaging to existing laboratory microscopes. "ALPHA3" is the hardware that extends the well documented benefits of light sheet imaging to existing laboratory microscopes, allowing rapid volume acquisition of a specimen. The light source gives wavelength options from $375 \mathrm{~nm}$ to $785 \mathrm{~nm}$; single or dual illumination arms; air or water lenses with finite to infinite options and an image sensor compatible with sCMOS cameras. The accompanying software and available optional software plus a MicroManager plug-in together with comprehensive acquisition options, facilitates automation. This system is also best suited for high throughput screening of conventionally prepared samples [30].

The mission statement of " $3 \mathrm{i}$ " (Intelligent Imaging Innovations) " is the provision of advanced multidimensional microscopy platforms that are intuitive to use, modular in design, and meet the evolving needs of investigators in the biological research community". They produce the "Lattice LightSheet microscope" invented by Eric Betzig of the Howard Hughes Medical Institute Janelia Research Campus, USA and also a dual-view Inverted Selective Plane Illumination (diSPIM) option. This same system is also marketed as the "American 3i Biological Microscope" (http://www.lind.cn/en/product/product-70-899.html). The Lattice LightSheet microscope is a modular LSFM system incorporating modular laser light sources offering a choice of 10 different illuminating wavelengths; 405, 445, 473, 488, 514, 532, 561, 594, 640 and 660nm. Laser firing is synchronized with data capture by the high speed high resolution sCMOS camera. This system is best suited to imaging living biological samples at subcellular resolution using time delayed 3D imaging. Every aspect of this system is controlled by SlideBook ${ }^{\mathrm{TM}} 6$ software including data capture, processing of raw image data [31].

"Cairn research" specializes in the supply of modular components for use in custom built LSFM units. This pragmatic approach reflects the economic reality facing instrument manufacturers with the possibility that an instrument may quickly be side-lined by other developments. This company specializes in the design and supply of customized solutions to meet customer requirements in this highly specialized and progressive field. They also supply software packages to fulfil the necessary data handling requirements of individual LSFM experiments. This software can be used to produce two dimensional image stacks from data collected over a particular time frame even when a range of illumination and detection wavelengths were used. Where a motorised $\mathrm{X}-\mathrm{Y}$ stage with $\mathrm{Z}$ focussing device is included as part of the modular custom built LSFM design, image stacks can be resolved in 5 dimensions [32]. Precision control of illumination sources, microscope stages, filters, camera etc. is provided for in a typical software program, so that it meets experimental requirements. The modular nature of their product range allows the biological researcher to custom design their LSF microscope to meet the exact conditions required for a particular experiment, and Cain supplied software will also meet the data handling requirements.

"Applied Scientific Instrumentation" offer three different LSFM options [33] a budget priced "Fixed Sheet system" that only allows manual adjustment of the sheet position with 2 manually adjusted objectives that must be then focused correctly on the light sheet by hand. They also offer a "Standard single-sided system" that has the Light sheet from one side and the emission objective on the other and a "Double-side system" that incorporates light sheet excitation and emission on both sides. The budget version has little versatility other than very basic applications and concerning the other versions they offer, the Standard single sided system although it has a straightforward set up that allows rapid scanning unfortunately achieves better XY resolution than $Z$ resolution. The best $\mathrm{X}, \mathrm{Y}$ and $\mathrm{Z}$ resolution is obtained from their Double-side system, but it is both expensive and complicated to use. They also supply suitable software for data handling.

"Luxendo" offer their "MuviSPIM", LSFM [34] which overcomes the need for sample rotation by achieving Multiview imaging from 4 simultaneous orthogonal views. This arrangement helps eliminate shadowing effects, while facilitating long term imaging and at a greatly increased speed of acquisition. The software they supply is in modular format and the stated aim of this flexible arrangement is to facilitate the design of complex experiments. The software incorporates real time fusion of captured 3D image data, which can then be streamed directly to the storage and data processing server that is designed to be a very efficient pipeline.

LSFM results in vast quantities of data being generated in any experiment which can be challenging to the finite storage 
resources of CPU's, and the development of compression algorithms offers the most efficient solution [35]. LSFM hardware is constructed to meet the sample requirements. Analytical software must then reconstruct the data and analyze it to answer the fundamental question being asked in an experiment. This involves use of specially designed algorithms capable of combining views of the sample recorded at different angles, fuse and de-convolute these images in 3D, thereby enabling both visualization and analysis of experimental data [36]. Software solutions are available from the various commercial sources already mentioned and also from the open source platforms allowing selection of software that compliments particular experimental data. Using these resources software can be obtained to meet the parameters of most LSFM experimental conditions. Data handling in LSFM is an area of intense research and the OpenSPIM platform offers extremely helpful advice to researchers, providing tried and trusted data management advice from basics such as ensuring "Loss-less or non-lossy compression" i.e. during image file size reduction, that the data stored is exactly the same as in the original. This is important as some compression files like JPEG (lossy) result in a certain amount of the image information being discarded [37] (Fiji, 2015). Ensuring that algorithms for data, processing to determine a particular threshold avoid subjective user decisions and are firmly based on image-intrinsic properties must be the basis of selection.

\section{CONCLUSION}

No imaging system capable of three dimensional resolution at a nanometer level is currently available that is ideal for all live cell imaging requirements. Every biologist must chose the microscope that best meets their needs based on:

1) The properties of the samples being examined.

2) The data they wish to acquire.

3) The data-load to be processed.

\section{STATEMENT}

The author is not affiliated to any commercial entity, and the views are a scholarly essay intended as an overview of some of the latest available options of this invaluable research tool.

\section{REFERENCES}

[1] Raleigh, L. (1879) 'Investigations in optics, with special reference to the spectroscope' Philosophical Magazine Series 5. 8 (49). pp 261-274. https://doi.org/10.1080/14786447908639684

[2] ISO standard ISO-21348 (2007)

[3] Higashiguchi, T. ; Otsuka, T. ; Yugami, N. ; Jiang, W. ; Endo, A. ; Dunne, P. ; Li, B.; O'Sullivan, G. (2011) 'Shorter-wavelength extremeUV sources below $10 \mathrm{~nm}$ ' SPIE Newsroom. DOI: 10.1117/2.1201109.003765

[4] Louis, E., Yakshin, A., Tsarfati, T., Bijkerk F. (2011) 'Nanometer interface and materials control for multilayer EUV-optical applications' Progress in Surface Science. Volume 86, Issues 11-12, December 2011, pp 255-294

https://doi.org/10.1016/j.progsurf.2011.08.001

[5] Anderson, E.H. (2006) 'Specialized electron beam nanolithography for EUV and X-ray diffractive optics' IEEE Journal of Quantum Electronics. 42(1), pp $27-35$
https://doi.org/10.1109/JQE.2005.858451

[6] Sattler, K. (2010) Handbook of Nanophysics: Nanoelectronics and Nanophotonics. CRC Press; Taylor \& Francis Group, Boca Raton, Florida USA. pp 19-13.

[7] Goldberg, K. ; Yashchuk, V.; Artemiev, N.; Celestre, R.; Chao, W. Gullikson, E.; Lacey, I.; McKinney, W.; Merthe, D.\& Padmore, H. (2013) 'Metrology for the advancement of X-Ray Optics at the ALS'Synchrontron Radiation News 26(5). pp 4-12.

[8] Wachulak P, Bartnik A, Fiedorowicz H. (2010) 'Sub-70 nm resolution tabletop microscopy at $13.8 \mathrm{~nm}$ using a compact laser-plasma EUV source'. Optics Letters; 35 (14). pp.2337-9. https://doi.org/10.1364/OL.35.002337

[9] Wachulak, P.; Bartnik, A \& Fiedorowicz, H. (2014) 'Extreme ultraviolet and soft x-ray microscopy using a compact gas puff target Laser-plasma source' Microscopy: advances in Scientific Research and Education,

(A Mendes - Villas,Ed.).

[10] Chao, W. ; Jihoon, K. ; Rekawa, S. ; Fischer, P. ; Anderson, E. H. (2009) 'Demonstration of $12 \mathrm{~nm}$ Resolution Fresnel Zone Plate Lens based Soft X-ray Microscopy’ Optics Express, 17 (20). p. 17669 https://doi.org/10.1364/OE.17.017669

[11] Artioukov, I. A.; Vinogradov, A. V.; Asadchikov, V. E.; Kas'yanov, Yu. S.; Serov, R. V.; Fedorenko, A. I.; Kondratenko, V. V.; Yulin, S. A. (1995) 'Schwarzschild soft-x-ray microscope for imaging of nonradiating objects'. Optics Letters, 20 (24), pp.2451-2453. https://doi.org/10.1364/OL.20.002451

[12] Wachulak, P. Bartnik, A, Fiedorowicz, H. Pánek, D. \& Brůža,P. (2012) 'Imaging of nanostructures with sub-100 nm spatial resolution using a desktop EUV microscope'. Applied Physics B 109 (1), pp 105-111 https://doi.org/10.1007/s00340-012-5125-3

[13] Wachulak, P.; Bartnik, A.; Fiedorowicz, H.\& Kostecki, J. (2011) 'A $50 \mathrm{~nm}$ spatial resolution EUV imaging-resolution dependence on object thickness and illumination bandwidth'. Optics Express 19(10), pp. 9541-9550. https://doi.org/10.1364/OE.19.009541

[14] Goldberg, K. (2012) 'Creating an EUV Mask Microscope for Lithography generations Reaching $8 \mathrm{~nm}$ ' in Proc. ASPE Precision Engineering and Mechatronics supporting the semiconductor industry, Berkeley, USA,4-7.

[15] Goldberg, K. (2011) 'SHARP: Advanced Extreme-Ultraviolet Microscopy for Developing Microchips' http://newscenter.lbl.gov/2011/10/28/sharp/

[16] Voie, A. H. Burns, D. H. \& Spelman, F. A. (1993) “Orthogonal-plane fluorescence optical sectioning: Three-dimensional imaging of macroscopic biological specimens" Journal of Microscopy 170 (3) pp. 229-236. https://doi.org/10.1111/j.1365-2818.1993.tb03346.x

[17] Huisken J, Swoger J, Del Bene F, Wittbrodt J\& Stelzer EH.(2004) "Optical sectioning deep inside live embryos by selective plane illumination microscopy." Science. 305(5686):1007-9. https://doi.org/10.1126/science.1100035

[18] Zanacchi, C.F, Lavagnino Z, Perrone Donnorso M, Del Bue A, Furia L, Faretta M, Diaspro A.(2011) "Live-cell 3D super-resolution imaging in thick biological samples" Nature Methods 8, 1047-1049 https://doi.org/10.1038/nmeth.1744

[19] Pastrana, E. (2013) "Bessel beams beyond the limit" Nature Methods $10,102-103$ https://doi.org/10.1038/nmeth.2354

[20] Gao,L, Shao, L, Chen, B-C. \& Betzig, E. (2014) "3D live fluorescence imaging of cellular dynamics using Bessel beam plane illumination microscopy" Nature Protocols 9, 1083-1101. https://doi.org/10.1038/nprot.2014.087

[21] Chen BC, Legant WR, Wang K, et al.(2014) 'Lattice light-sheet microscopy: imaging molecules to embryos at high spatiotemporal resolution'.Science.346(6208):1257998.doi:10.1126/science.1257998. https://doi.org/10.1126/science.1257998

[22] Pitrone, P.G, Schindelin, J. Stuyvenberg, L., Preibisch, S. Weber, M, Eliceiri,K.W. , Huisken, J.\& Tomancak, P. (2013) Nature Methods 10, 598-599. https://doi.org/10.1038/nmeth.2507

[23] Picard (2015) “USB 4D-STAGE” Picard Industries, Albion, NY, USA.

[24] Gualda, E.J, Vale,T. Almada, P, Feijó, J.A, Martins, G. \& Moreno, N. (2013)" Open Spin Microscopy: an open-source integrated microscopy platform" Nature Methods 10, 599-600. https://doi.org/10.1038/nmeth.2508 
[25] Huisken J, Swoger J, Del Bene F, Wittbrodt J\& Stelzer EH.(2004) "Optical sectioning deep inside live embryos by selective plane illumination microscopy." Science; 305(5686, pp.1007-9. https://doi.org/10.1126/science.1100035

[26] Lavisionbiotec, (2015) "UltraMicroscope II The Light Sheet Microscope" http://www.lavisionbiotec.com/ultramicroscopedescription.html

[27] Dodt HU, Leischner U, Schierloh A, Jährling N, Mauch CP, Deininger K, Deussing JM, Eder M, Zieglgänsberger W, Becker K.(2007) "Ultramicroscopy: three-dimensional visualization of neuronal networks in the whole mouse brain". Nature Methods 4, 331 - 336. https://doi.org/10.1038/nmeth1036

[28] Leica, (2015) "The Vertical Turn- Combining Light Sheet and Confocal Leica TCS SP8 DLS." Genoa. http://www.lsfm2015.org/img/lsfm-2015book.pdf http://www.leica-microsystems.com/products/confocalmicroscopes/details/product/leica-tcs-sp8-dls/

[29] ASlimaging, (2014). 'Selective plane microscopy'. http://www.asiimaging.com/

[30] Phaseview (2015) "Turn your microscope in a powerful light sheet imaging platform" http://phaseview.com/3d-digital-imaging/alpha3/

[31] 3 i (2015) "Lattice Light Sheet" https://www.intelligentimaging.com/systems.php

[32] Cairn, (2015) "LSFM Products" http://www.cairnresearch.co.uk/products/software/fluorescence-software

[33] ASIimaging.(2014) 'Selective plane microscopy.' http://www.asiimaging.com/

[34] LUXENDO GmbH, (2015) "The Microscope SEEING LIFE FROM A DIFFERENT ANGLE - OR FOUR" http://luxendo.eu/

[35] Reynaud, E.G. ,Peychl, J., Huisken, J. \& Tomancak, P.(2015) "Guide to light-sheet microscopy for adventurous biologists." Nature Methods 12, pp. 30-34.

https://doi.org/10.1038/nmeth.3222

[36] Preibisch, S., Amat, F., Stamataki, E., Sarov, M., et al.(2014). "Efficient Bayesian-based multiview deconvolution." Nature Methods, 11(6), 645-648.

https://doi.org/10.1038/nmeth.2929

[37] Fiji. (2015). Image processing principles. http://fiji.sc/Principles 